\title{
A formula for the error of finite sinc-interpolation over a finite interval
}

\author{
Jean-Paul Berrut
}

Received: 18 December 2006 / Accepted: 22 February 2007 /

Published online: 10 May 2007

(C) Springer Science + Business Media B.V. 2007

\begin{abstract}
Sinc-interpolation is a very efficient infinitely differentiable approximation scheme from equidistant data on the infinite line. It, however, requires that the interpolated function decreases rapidly or is periodic. We give an error formula for the case where neither of these conditions is satisfied.
\end{abstract}

Keywords Sinc-interpolation - Infinitely differentiable approximation scheme - Equidistant interpolation on the interval • Error formula

\section{Introduction}

Approximating a function $f$ from a sample at equidistant abscissae is a classical problem in engineering that gives rise to interesting mathematics and recurrently leads to the publication of survey papers and books such as $[4,5,8,9,15]$. One approach to Shannon's sampling theory takes advantage of the Lagrange property

$$
S(k \pi)=\left\{\begin{array}{l}
1, k=0, \\
0, k \in \mathbb{Z} \backslash\{0\},
\end{array}\right.
$$

In celebration of Leonhard Euler's 300th birthday.

Work partly supported by the Swiss National Science Foundation under grant Nr 200020-103662/1.

J.-P. Berrut $(\bowtie)$

Département de Mathématiques, Université de Fribourg Pérolles, $\mathrm{CH}-1700$ Fribourg, Switzerland e-mail: jean-paul.berrut@unifr.ch 
of the sinc function

$$
S(x):=\frac{\sin x}{x}
$$

at the integer multiples of $\pi$ to construct the cardinal interpolant

$$
C(f, h)(x):=\sum_{n=-\infty}^{\infty} S\left[\frac{\pi}{h}\left(x-x_{n}\right)\right] f_{n}
$$

from the sample values $f_{n}:=f\left(x_{n}\right)$ at the bi-infinite sequence of equidistant arguments $x_{n}=n h$ (thus including zero). If $f$ decays rapidly enough at infinity, Shannon's sampling theorem asserts that $C(f, h)=f$ for $h$ sufficiently small if $f$ is the restriction to $\mathbb{R}$ of a function of exponential type (Paley-Wiener class, [13], p. $22 \mathrm{ff}$.), while one has exponential convergence of $C(f, h)$ when $f$ is analytic in a horizontal strip about $\mathbb{R}$ ([13], p. 35 or [17], p. 136). These facts make $C(f, h)$ unarguably the most important infinitely differentiable interpolant between equidistant points on the infinite line and on the circle, where it is the trigonometric interpolant [1].

When applying (1.1) in practice, one must restrict oneself to finite sums, which we will take here symmetric about 0 and make longer and longer to improve accuracy. This may be done in at least two ways (and their combinations): one may increase the number of terms for a fixed $h$; alternatively, and this will be our approach here, one may take a fixed interval $[-X, X]$ and approximate $C(f, h)$ of $(1.1)$ with the finite interpolant

$$
C_{N}(f, h)(x):=\sum_{n=-N}^{N} S\left[\frac{\pi}{h}\left(x-x_{n}\right)\right] f_{n}, \quad h=\frac{X}{N} .
$$

We are interested in the error $C_{N}(f, h)-f$ as a function of $h$ for $f \in C^{\infty}(\mathbb{R})$ (in fact, since we work only on a fixed interval, $f \in C^{\infty}[-X, X]$ is sufficient). Then one has $C_{N}(f, h)=C(\widetilde{f}, h)$ with

$$
\tilde{f}(x):= \begin{cases}f(x), & \text { if }|x| \leq X, \\ 0, & \text { otherwise }\end{cases}
$$

To avoid the discontinuities at $\pm X$, many authors, among them de la Vallée Poussin [18], assume that $f$ vanishes outside the interval under consideration, i.e., that the support of $f$ belongs to $(-X, X)$. Here we will be concerned with arbitrary functions $f \in C^{\infty}(\mathbb{R})$, thus also with those for which $\widetilde{f}$ has a jump at $X$ and $-X$. To diminish the impact of the jump, and in the spirit of Fourier series (see also [3], p. 95), we will replace the values $\widetilde{f}( \pm X)$ by the average values at the corresponding jumps, i.e., we will interpolate the following function, again denoted by $f$ :

$$
f(x):= \begin{cases}\widetilde{f}(x), & \text { if }|x|<X, \\ \widetilde{f}(x) / 2, & \text { otherwise. }\end{cases}
$$


Our interpolant $C_{N}(f, h)$ thus is the same as (1.2), up to the first and last terms which will be halved, an operation marked by a double prime appended to the sum.

\section{Preliminaries on numerical quadrature}

A classical formula for estimating the integral $I:=\int_{0}^{X} f(y) d y$ from an equispaced sample $f_{n}:=f\left(x_{n}\right)$ of $f$ at $x_{n}:=n h, h:=\frac{X}{N}$, is the trapezoidal rule $T_{h}(I):=h \sum_{n=0}^{N} f_{n}$, whose error is given by the Euler-Maclaurin formula

$$
T_{h}(I)-I=\sum_{k=1}^{\infty} a_{2 k} h^{2 k}, \quad a_{2 k}:=\frac{B_{2 k}}{(2 k) !}\left[f^{(2 k-1)}(X)-f^{(2 k-1)}(0)\right],
$$

where the constants $B_{2 k}$ are the Bernoulli numbers $[2,16]$. The series-notation " $\infty "$

$\sum$ in (2.1) does not presume anything about convergence, it just expresses the fact that there is no limit to $k$ when $f \in C^{\infty}$ (in fact, the series does not usually converge). If the highest even order of differentiability of $f$ is $2 m+2$, then the error becomes $\sum_{k=1}^{m} a_{2 k} h^{2 k}+\mathcal{O}\left(h^{2 m+2}\right)[11] . \sum_{k=1}^{\infty} c_{2 k} h^{2 k}$ will here simply mean $\sum_{k=1}^{m} c_{2 k} h^{2 k}+\mathcal{O}\left(h^{2 m+2}\right)$ for every $m \in \mathbb{N}$.

If the abscissae do not include the endpoints, as with $x_{n+\frac{1}{2}}:=\left(n+\frac{1}{2}\right) h, n=$ $0, \ldots, N-1$, then one possibility is the midpoint rule $M_{h}(I):=h \cdot \sum_{n=0}^{N-1} f_{n+\frac{1}{2}}$, whose error is

$$
M_{h}(I)-I=-\sum_{k=1}^{\text {“'” }}\left(1-2^{1-2 k}\right) a_{2 k} h^{2 k}
$$

with the same $a_{2 k}$ as in (2.1) ([6], p. 139).

Euler-Maclaurin formulae have been given for Cauchy principal value integrals $I_{x}:=\operatorname{PV} \int_{0}^{X} \frac{f(y)}{x-y} d y$ as well. Restricting to analytic $f$ 's, Hunter [10] has subtracted an analytically integrable $X$-periodic function with the behavior of $\frac{1}{x-y}$ at $x$, and so that the endpoint values of each of its derivatives coincide, to obtain the formula

$$
\begin{aligned}
& T_{h}\left(I_{x}\right)=h \sum_{n=0}^{N "} \frac{f_{n}}{x-x_{n}}=I_{x}+\pi f(x) \cot \frac{\pi}{h} x+\sum_{k=1}^{\infty} a_{2 k}(x) h^{2 k}, \\
& a_{2 k}(x):=\frac{B_{2 k}}{(2 k) !}\left[\left(\frac{f(y)}{x-y}\right)^{(2 k-1)}(X)-\left(\frac{f(y)}{x-y}\right)^{(2 k-1)}(0)\right] .
\end{aligned}
$$

(Hunter gives also formulae for the case when $x$ is one of the sample abscissae, but this will not occur in our context.) Lyness [14] later noticed that the subtraction function could be simply taken as $\frac{\pi}{X} f(x) \cot \left[\frac{\pi}{X}(x-y)\right]$ and gave 
the $\mathcal{O}\left(h^{2 m+1}\right)$ term for $f \in C^{2 m+1}$; Elliott [7] fixed a slight error in Lyness' assumptions. Hunter also gave the corresponding formula for the midpoint rule as

$$
M_{h}\left(I_{x}\right)=h \sum_{n=0}^{N-1} \frac{f_{n+\frac{1}{2}}}{x-x_{n+\frac{1}{2}}}=I_{x}-\pi f(x) \tan \frac{\pi}{h} x-\sum_{k=1}^{\infty}\left(1-2^{1-2 k}\right) a_{2 k}(x) h^{2 k}
$$

with the $a_{2 k}$ of (2.3). (Our signs do not match those of [10,14] or [7] for we integrate $\frac{f(y)}{x-y}$ instead of $\frac{f(y)}{y-x}$, which changes all signs, including that of the residue in [10].)

We will be concerned with formulae for symmetric integrals $\mathrm{PV} \int_{-X}^{X} \frac{f(y)}{x-y} d y$. Since the singular point is either in $[-X, 0]$ or in $[0, X]$ and if $N$ is even, one may simply add formula (2.1) and (2.3), resp. (2.2) and (2.4), for $\frac{N}{2}$ to see that (2.3) and (2.4) hold with 0 replaced by $-X$ in $a_{2 k}$. (Another way of seeing the same: if $x \in[-X, 0]$, the change of variable $y=z-X$ does not modify the factor $\cot \frac{\pi}{h} x$ in the derivative-free term in view of the $\pi$-periodicity of the cotangent.)

However, this is not true when $N$ is odd, as 0 is no longer an integration node of the trapezoidal rule. That same change of variable yields $\mathrm{PV} \int_{-X}^{X} \frac{f(y)}{x-y} d y=$ PV $\int_{0}^{2 X} \frac{f(z-X)}{x+X-z} d z$ and, in view of $X=\frac{N}{2} h$, the derivative-free term becomes $\pi f(x) \cot \left[\frac{\pi}{h}(x+X)\right]=-\pi f(x) \tan \frac{\pi}{h} x$ in (2.3) and $-\pi f(x) \tan \left[\frac{\pi}{h}(x+X)\right]=$ $\pi f(x) \cot \left[\frac{\pi}{h}(x)\right]$ in (2.4): these terms exchange place in the formulae.

\section{The error formula}

We now turn to our aim, namely that of finding a formula for $C_{N}(f, h)-f$ for $f$ in (1.3). One immediately sees that $C_{N}(f, h)$ may be written as $[1,12,18]$

$$
C_{N}(f, h)(x)=\frac{h}{\pi} \sin \frac{\pi}{h} x \sum_{n=-N}^{N} "(-1)^{n} \frac{f_{n}}{x-x_{n}} .
$$

The right-hand expression may be interpreted as a difference of quadrature formulae of Section 2 with step $2 h$ :

$$
\begin{aligned}
C_{N}(f, h)(x) & =\frac{1}{2 \pi} \sin \left(\frac{\pi}{h} x\right)(-1)^{N}\left(T_{2 h}\left(I_{x}\right)-M_{2 h}\left(I_{x}\right)\right), \\
I_{x} & =\operatorname{PV} \int_{-X}^{X} \frac{f(y)}{x-y} d y
\end{aligned}
$$


(the factor $(-1)^{N}$ cares for the sign at the extremal nodes in dependence on the parity of $N$ ). But, with $\widetilde{h}:=2 h$ and if $N$ is even so that $T_{2 h}$ and $M_{2 h}$ cover an even number of intervals,

$$
\begin{gathered}
T_{\widetilde{h}}\left(I_{x}\right)=I_{x}+\pi f(x) \cot \frac{\pi}{\widetilde{h}} x+\sum_{k=1}^{\infty} a_{2 k}(x) \widetilde{h}^{2 k}, \\
M_{\widetilde{h}}\left(I_{x}\right)=I_{x}-\pi f(x) \tan \frac{\pi}{\widetilde{h}} x-\sum_{k=1}^{\infty}\left(1-2^{1-2 k}\right) a_{2 k}(x) \widetilde{h}^{2 k} .
\end{gathered}
$$

For odd $N$ the exchange of the derivative-free terms in $T_{2 h}$ and $M_{2 h}$ introduces another factor $(-1)^{N}$ in their difference.

Subtracting, using the trigonometric identity $\tan \alpha+\cot \alpha=2 / \sin 2 \alpha$, and simplifying yield the first version of the formula alluded to in the title,

$$
\begin{aligned}
C_{N}(f, h)(x) & =f(x)+\frac{(-1)^{N}}{2 \pi} \sin \frac{2 \pi}{\widetilde{h}} x \sum_{k=1}^{\infty} b_{2 k}(x) \widetilde{h}^{2 k}, \\
b_{2 k}(x) & :=2\left(1-2^{-2 k}\right) a_{2 k}(x),
\end{aligned}
$$

where the $a_{2 k}(x)$ are given by (2.3) with 0 replaced by $-X$.

The oscillatory sine-factor in front of the sum vanishes at every $x_{n}$, reflecting the interpolation property. On the other hand, it complicates the practical interpretation of the formula. For a given $x$ and a fixed number $P$ of terms in the sum, it is namely possible to pick a sequence of $h$ for which the factor is growing toward 1 from a value close to 0 . To avoid this, one may take a factor $h$ in front of the sine to get

$$
C_{N}(f, h)(x)=f(x)+(-1)^{N} x S\left(\frac{2 \pi}{\widetilde{h}} x\right) \sum_{k=1}^{\infty} b_{2 k}(x) \tilde{h}^{2 k-1} .
$$

Now, as $h \rightarrow 0$, since $S\left(\frac{2 \pi}{h} x\right) \downarrow 0$, the above effect, though not to be ignored for small $P$, disappears asymptotically.

Summarizing we have the following.

Theorem 3.1 Let $f \in C^{\infty}[-X, X]$ be interpolated on the interval $[-X, X]$ by the sinc-interpolant $C_{N}(f, h)$ in (3.1) with $N \in \mathbb{N}, h=\frac{X}{N}$ and $x_{n}=n h$. Then the difference $C_{N}(f, h)-f$ is given by formula (3.3) or, equivalently, (3.4).

The extended version of this paper will contain the corresponding formulae for the more involved case of an even number of interpolation points. In the study by Berrut (First applications of a formula for the error of finite sincinterpolation, in preparation) we will give first applications. 


\section{References}

1. Berrut, J.-P.: Barycentric formulae for cardinal (SINC-)Interpolants. Numer. Math. 54, 703-718 (1989) [Erratum 55, 747 (1989)]

2. Berrut, J.-P.: A circular interpretation of the Euler-Maclaurin formula. J. Comput. Appl. Math. 189, 375-386 (2006)

3. Briggs, W.L., Henson, V.E.: The DFT: An Owner's Manual for the Discrete Fourier Transform. SIAM, Philadelphia (1995)

4. Butzer, P.L., Splettstösser, W., Stens, R.L.: The sampling theorem and linear prediction in signal analysis. Jahresber. Dtsch. Math.-Ver. 90, 1-70 (1988)

5. Butzer, P.L., Stens, R.L.: Sampling theory for not necessarily band-limited functions: a historical overview. SIAM Rev. 34, 40-53 (1992)

6. Davis, P.J., Rabinowitz, P.: Methods of Numerical Integration, 2nd edn. Academic Press, San Diego, CA (1984)

7. Elliott, D.: The Euler-Maclaurin formula revisited. J. Aust. Math. Soc. B 40, E27-E76 (1998) (Electronic)

8. Higgins, J.R.: Five short stories about the cardinal series. Bull. Am. Math. Soc. 12, 45-89 (1985)

9. Higgins, J.R.: Sampling Theory in Fourier and Signal Analysis, Foundations. Clarendon, Oxford (1996)

10. Hunter, D.B.: The numerical evaluation of Cauchy principal values of integrals by Romberg integration. Numer. Math. 21, 185-192 (1973)

11. Kincaid, D., Cheney, W.: Numerical Analysis, Mathematics of Scientific Computing. Wadsworth, Belmont (1991)

12. Kress, R.: Interpolation auf einem unendlichen Intervall. Computing 6, 274-288 (1970)

13. Lund, J., Bowers, K.L.: Sinc Methods for Quadrature and Differential Equations. SIAM, Philadelphia (1992)

14. Lyness, J.N.: The Euler Maclaurin expansion for the Cauchy principal value integral. Numer. Math. 46, 611-622 (1985)

15. Partington, J.R.: Interpolation, Identification and Sampling. Clarendon, Oxford (1997)

16. Schwarz, H.R.: Numerische Mathematik, 4te Aufl., Teubner, 1997; English translation of the 2nd edn: Numerical Analysis. A Comprehensive Introduction. Wiley, New York (1989)

17. Stenger, F.: Numerical Methods Based on Sinc and Analytic Functions. Springer, Berlin (1993)

18. de la Vallée Poussin, C.J.: Sur la convergence des formules d'interpolation entre ordonnées équidistantes. Bull. Cl. Sci. Acad. R. Belg. Série 4, 319-410 (1908) 